\title{
Pressure development in a non-Newtonian flow through a tapered tube
}

By S. Oka (Yokohama)

Rheol. Acta 12, 140-143 (1973)

$\begin{aligned} & \text { p. 141, Eq. [16], } \\ & \text { read correctly: }\end{aligned} \frac{\partial}{\partial r}\left[\frac{1}{r} \frac{\partial}{\partial r}\left(\eta_{a} r \frac{\partial u}{\partial r}\right)\right]=0$.

\section{Comportement des matériaux plastiques parfaits, non visqueus}

Par M. Hajal (Beyrouth)

Rheol. Acta 12, 150-155 (1973)

p. 150, Notations,

please, add after $E_{2}$ :

$E_{3}=\left(e_{i j} e_{j k} e_{k i}\right)^{1 / 3}$

p. 151, Eq. [3], second line read correctly:

$s_{i j}=-\frac{c}{3} \delta_{i j} \ldots$

Eq. [4], second line read correctly:

$s_{i j}=-\frac{c}{3} \delta_{i j} \ldots$

p. 152, left column, second eq. at the head read correctly:

$\cos \theta=s_{i j / s_{2} i j / E_{2}}$ right column, Hypothèse 3, b, first eq. read correctly:

$$
m / E_{2}=p=\frac{1}{\sqrt{3}}
$$

p. 155, add the following English Summary:

Summary

In the first part, starting from a general stress-strain rate and specific weight relationship, we have proved the existence of a yield surface (relation between stresses). In the second part, we have proposed a flow rule. As special cases of that flow rule we find $=$ the plastic potential theory for incompressible materials with a yield surface independant of the mean stress, the theory of Brown and Gudehus for compressible materials with a yield surface independent of mean stress, and the theory of intrinsic curve (shearing along a plane) for materials with a yield surface independent of intermediate principal stress.

\section{An extension of Reiner's "Deborah Number" concept to a wide field of rheological investigations}

By G. W. Scott Blair (Iffley, Oxford)

Rheol. Acta 12, 235-236 (1973)

p. 235, second paragraph, left column, first sentence:

Reiner wrote his number with the Hebrew letter "dalet", which could not be given in print. Instead of $T$, please, read "dalet"! 